\title{
The Growth rate Tracking System of the Tree for Forest industry
}

\author{
Nimit Hongyim*, Somsak Mitatha \\ Department of Computer Engineering, Faculty of Engineering \\ King Mongkut's Institute of Technology Ladkrabang, \\ Bangkok 10520, Thailand.
}

*Corresponding Author: nimith@siamsq.net

\begin{abstract}
This paper described on how to use the Automate system for Growth Rate tracking of each tree in the forest industry which will help to solve the problem to predict the cycle time of each tree will be grown after previous batch of tree has been cut down for forest industry. System designed to keep track of Tree body when it expand to larger and Flex sensor used to wrap around detected the changing while the tree body is getting larger and the percent of the expand of tree body will convert to be growth rate then send them to database server wirelessly both short distance between tree to outpost station by using X-Bee and long length from outpost to Gateway station where more than 1-3 Km away by using Packet Radio or APRS protocol. The growth rate data will use to create the profile of growth rate which will use to forecasting of wood production consist of the timeline of each batch in the next plantation of new trees.
\end{abstract}

Keywords: Flex Sensor, Packet Radio, APRS, X-Bee module,

\section{Introduction}

In the forest industry, there is usually no record of growth rate of each type of trees. In the forest industry where is in a wide area and used labor to take note the growth rate of tree seedlings from the trees mature till it can be cut to be processed into finished wood that will be very difficult and requires a lot of labors and time to taking note which led to the growth plans for planting and cutting area. This Problem will solved by designing automation systems that keep track the growth rate by using Flex sensors. Sensor warp around the trunk of the tree then measuring the growth rate since from seedling till the trunk is getting larger and that the characteristic of Flex Sensor which wrap around the trunk change angle which translate to result of resistance to the form of bent by the change in the rate of bending to voltage divider schematic which will feed in to Analog to Digital will be entered to Microcontroller for processing and sending the converted value of the growth rate of the period and the value of each local station to receive data using $\mathrm{X}-\mathrm{Bee}$, and will then communicate with the database server. By using data transmission to remote offices or stations connected to the network by sending data through the use of Radio Frequency in VHF $144.390 \mathrm{MHz}$ by this Packet radio or APRS protocol ${ }^{(1-3)}$ could have transmission distance of 1-2 kilometers away which this protocol is the most stable for long length data communication for the instant that also used for Satellite communication that is the reason why Packet Radio or APRS is the right choice for protocol to send data from rural area to gateway station that connected to main network system.

\section{Design Concept}

\subsection{Block Diagram}

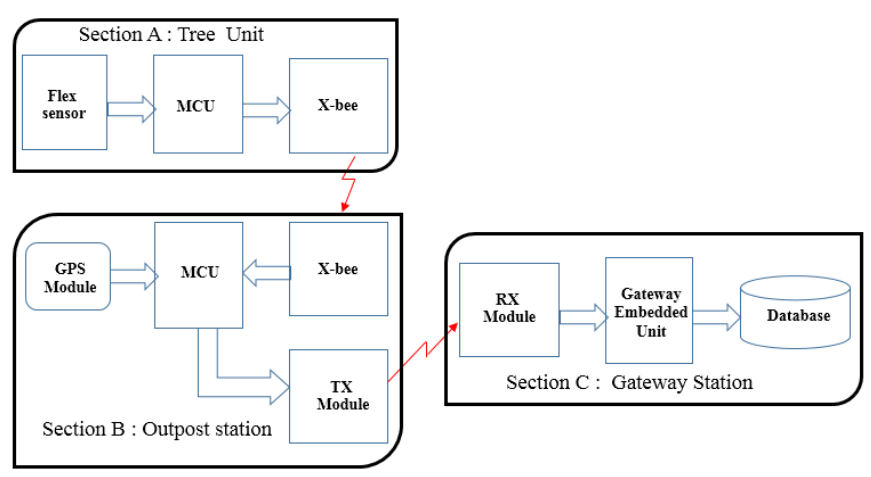

Fig. 1. Block Diagram of system 
The system will consist of 3 sections

a. Tree unit with Flex Sensor and X-Bee

b. Outpost station X-Bee and RF-TX Module

c. Gateway station RX-Module and Embedded System with network connection to Database Server

In the System as on Fig.1. We planned to have many of Tree stations which send data of each tree to Outpost station by using X-Bee which have limited of length of communication then the Outpost collected all data and send them all to Gateway station by using Radio TX Module via VHF band $144.390 \mathrm{MHz} 300 \mathrm{~mW}$ or more depend on the geographic of forest area.

\section{(a) Tree Unit:}

Each tree have Flex sensor which integrated by wrap around truck of tree the flexible indication detected by Flex Sensor and processed the data by MCU then send data by using X-Bee to outpost station

\section{- $\quad$ Flex Sensor:}

In the fig.2. Shows 4.5" length of Flex sensor, this sensor is a Resistance variable strip that resistance varied when strip is flexed

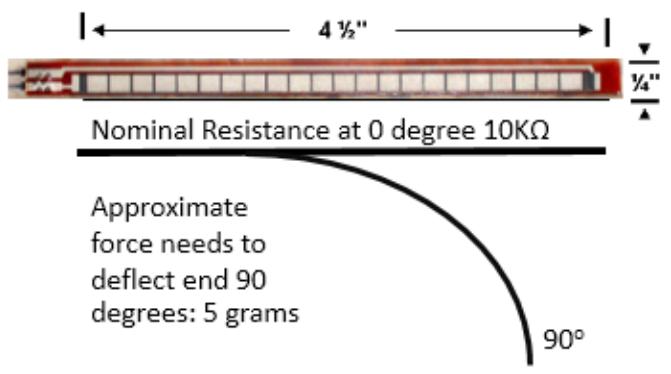

Approximate Resistance at 90 degree $14 \mathrm{~K} \Omega$ Approximate Resistance at 180 degree $22 \mathrm{~K} \Omega$

Fig. 2. Flex Sensor ${ }^{(4)}$

The flex sensor has a typical electrical resistance variation when flexed or bent as shown in Fig. 3.

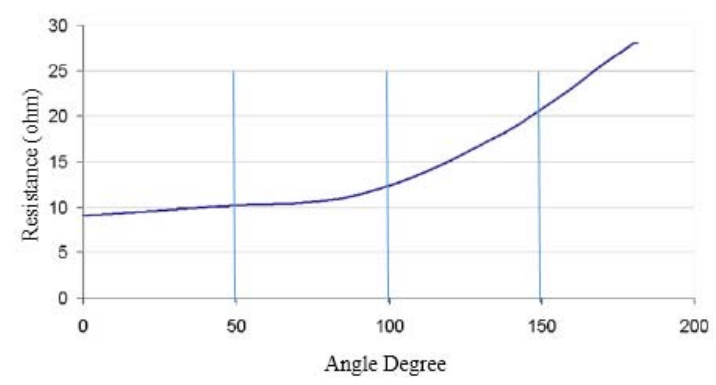

Fig. 3. Flex Sensor ${ }^{(5)}$
In each degree which will transform to voltage by using voltage divider

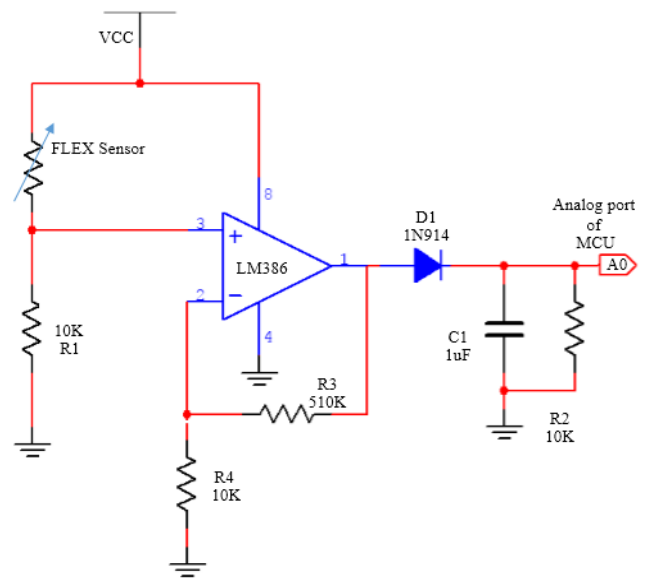

Fig. 4. Flex sensor amplification and filtration circuit ${ }^{(6)}$

Equations of non-inverting op-amp configuration to apply gain to our signal. The op-amp has rail voltages of $5 \mathrm{~V}$ and ground. The gain for the circuit is given by:

$$
A_{V}=1+\frac{R 2}{R 1}
$$

The circuit will saturate the op-amp at the top rail voltage and trigger a rising edge strong enough to trigger the microcontroller. After applying this gain, then send the input through a diode to clean the signal of any negative voltages, and removing any voltages under the diode's turn-on voltage then use a capacitor and resistor in parallel to have a sample holder. This sample holder acts as a filter for the signal ${ }^{(6)}$

A resistance value of $10 \mathrm{~K} \Omega$ matches an angle of $0^{\circ}$ while a value of $14 \mathrm{~K} \Omega$ will matches an angle of $90^{\circ}$ and a value of $22 \mathrm{k} \Omega$ matches an angle of $180^{\circ}$. Data on the mapping between the resistance and angles is supplied by the sensor manufacturer. For the resistance value obtained from the flex sensor, by using interpolation equations, it is possible to calculate the corresponding bent angle.

To compute the value of the resistance from the Flex sensor one uses the voltage divider formula, as follows ${ }^{(7)}$

$$
R_{\text {Flex sensor }}=\frac{R_{1}\left(V_{\text {in }}-V_{\text {out }}\right)}{V_{\text {out }}}
$$

$R_{\text {Flex sensor }}=$ the resistance value

$\underline{R}_{1}=10 \mathrm{k} \Omega, \quad \operatorname{Vin}=\operatorname{Vcc}(5 \mathrm{~V})$,

$V_{\text {out }}$ is the voltage value which connect to ADC pin 
For the conversion of the resistance value to the angle value two formulas were used ${ }^{(7)}$

$$
\begin{gathered}
\theta_{1}\left[^{\circ}\right]=\frac{R_{\text {Flex sensor }}}{44.44} \\
\theta_{2}\left[^{\circ}\right]=\frac{R_{\text {Flex sensor }}-6001}{88.88}
\end{gathered}
$$

Where $\theta_{1}$ and $\theta_{2}$ are the angles for the corresponding flex sensor resistance value. Equation (3) is used when the resistance value is between $10 \mathrm{k} \Omega$ and $14 \mathrm{k} \Omega$ while equation (4) is used when the resistance value is between $14 \mathrm{k} \Omega$ and $22 \mathrm{k} \Omega$.

\section{- $\quad$ X-BEE module $(8-10)$}

IEEE 802.15.4 suite for high level communication which used to create small personal network with low power radio signal which the deformation angles are collected and data is transmitted across the IEEE 802.15.4 network.

\section{$-\quad \mathrm{MCU}$}

The high-performance Atmel ATMEGA328P (11) Pico-Power 8-bit AVR RISC-based Microcontroller, a 6-channel 10-bit A/D converter are programmed to sampling the value of voltage that came from Flex Sensor

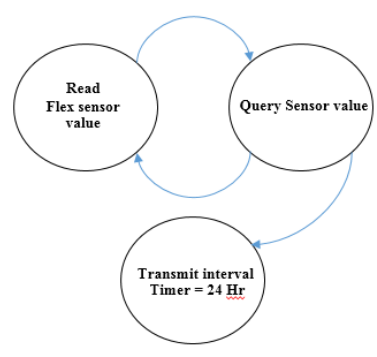

Fig. 5. Flow chart of Tree Unit

\section{(b) Outpost Station}

This main function of this section, MCU which same type as in the Tree unit programed to query data from $\mathrm{X}$-Bee which act as the receiver from each Tree unit then combine them together with the GPS data which installed and it will help to indicate the area of the lot and then we can use this coordinate where we can put the location overlay on the Google map after query from Database server. MCU will process all the data of the Flex sensor which send over to the IEEE 802.15.4 network then combined with GPS data then encoded to the Packet Radio or APRS format then convert them to AFSK (Audio Frequency Shift Keying) send over Radio Frequency. This research is using 1200 bps of data communication speed over the air by Radio Matrix Module ${ }^{(12)}$ for Narrow band VHF $144.390 \mathrm{MHz}$ by using small antenna for TX $(300 \mathrm{~mW})$ and used the small Half-wave Antenna, expected to be able for communication range at $1-2 \mathrm{Km}$ in rural/open (line of sight) to the receive station as known as Gateway Station

\section{(c) Gateway Station}

This section consist of the Receiver unit which used for receive the Packet Radio which sent over VHF 144.390MHz and then feed to I-Gate Microsat ${ }^{@}$ Embedded Unit ${ }^{(13)}$ which the unit consist of the Packet Radio Decoder with DSP capability and Network connectivity which will processed decoded packet and then send to APRS-IS (APRS Internet Server) port 14580 via Internet Network. All the data will be in the APRS-IS ${ }^{(14)}$ and then we can query raw data that can be put overlay to Google Map open API or display in graph format.

\section{Infrastructure}

\subsection{Tree Unit Installation}

In order to install Flex sensor it will need to understand the type of tree that we used in this research, in the forest industry in this paper was done by using data from the research paper that described and correct data of Eucalyptus Plantation for 2-4 years and average diameter was 4.8 inches and average height was 51 feet. But for the forest wood industry only interested in diameter that expected at maximum size at 4 inches that considered good for cutting to sending to wood industry ${ }^{(15)}$

Flex Sensor condition according to the growth rate as which angle represented as percent of fully grow as expected 4" approximately 100\% shown in Fig 6 


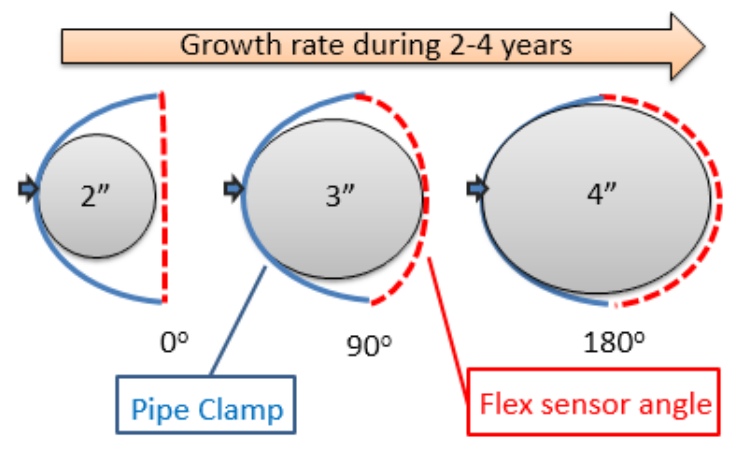

Fig. 6. Flex Sensor Condition with Growth Rate

The sensor installation was mounting by using PVC Pipe clamping and Flex Sensor as shown in Fig 7

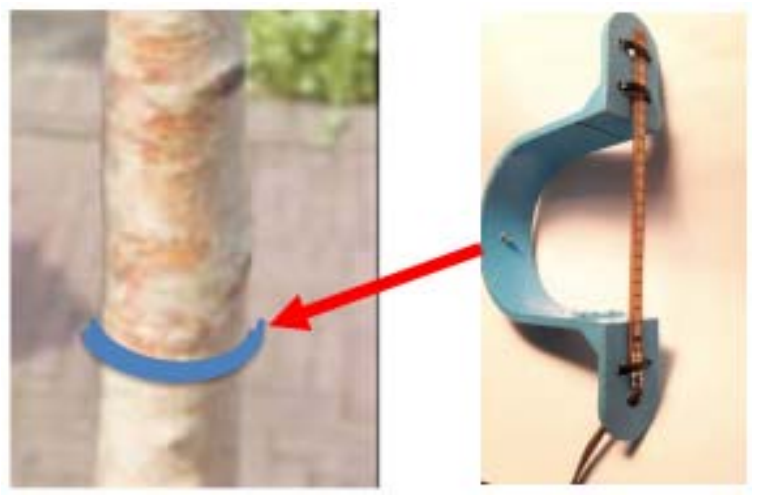

Fig. 7. Flex sensor Mouthing

\subsection{Infrastructure of the system}

The concept of this paper by deploy the Tree unit as sample size per area that will detect the change of the growth rate with unit ID (Tree\#x) send to the Outpost station which will collect all the tree units then send out the summary of all the trees that include in that field area in the same time along with GPS data then encode to APRS or Packet Radio then send in RF on VHF band $144.390 \mathrm{MHz}$ to the Gateway station which equipped with Receiver, Encoder and Embedded that capable to feed all the data to the APRS.IS(database server) and then the Web interface will be able to query data back then process in whatever need to analyze or planning forecast later as in Fig.8.

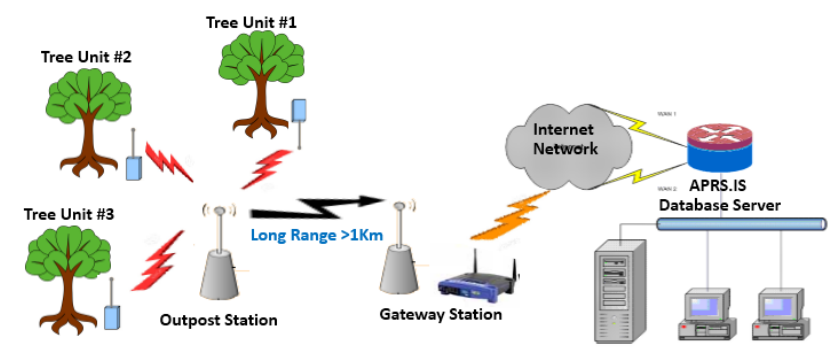

Fig. 8 Infrastructure of system

\section{Field Test}

Tree unit were installed in 3 Trees by using Flex sensor mouthing as shown in Fig 7. around Eucalyptus Plantation selected Tree age 2 years old when the tree diameter begin at 2 inches which will expand to maximum at 4 inches in another 2 years. At this point the unit sent the sample data to Outpost station and the data was sent by outpost station fed to the Gateway and the Web query interface ${ }^{(16)}$ were done by using the sample API overlay Google Map. We can see the detail of description as shown in the message box over the location which done by GPS data came from GPS unit installed in the Outpost unit which shown in Fig.9

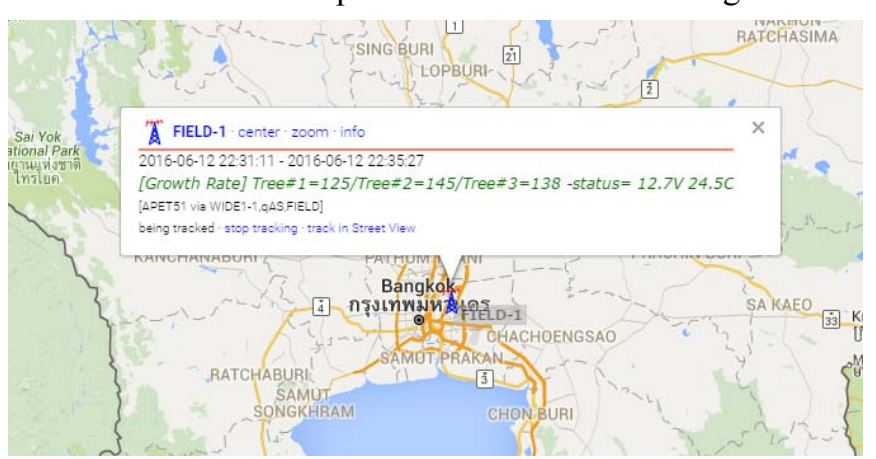

Fig. 9. Web interface online Map

In order to send Packet to Standard APRS Server, Data format need to follow as in the Table Lat / Long Position Reports are contained in the Information field and the Flex Sensor reading data assigned to Comment field of an APRS AX.25 frame ${ }^{(17)}$. As shown in Fig.10. Raw packets were decoded by Gateway station which use embedded system.

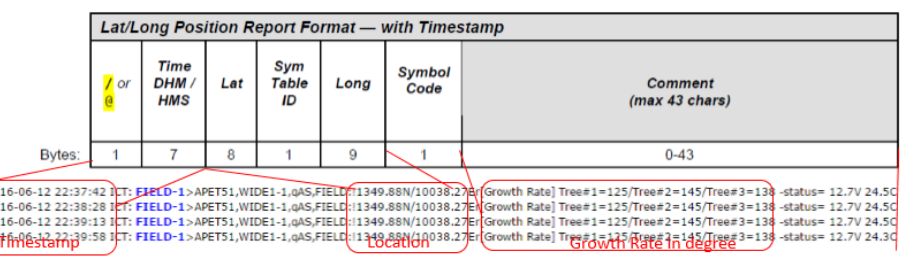

Fig. 10. RAW data send to database server

\section{Result}

The result of this paper was done in the rural area as the Forest Industrial located in the east of Bangkok, Thailand. Installed 3 Tree Units but because the fact that Tree growth rate will not grow as fast to get the real result because it will take another 2-4 years from now but we did simulation the angle data in order to send the angle data from Tree unit of Flex sensor measurement from each tree to Outpost 
station to the database server by using wireless communication which sending by X-Bee module in $2.4 \mathrm{GHz}$ RF carrier in very short distance data then from Outpost station combined data of 3 Tree units and then sent Packet data to the Gateway station location where located over 2 Km away by using VHF TX module 144.390MHz 300mW transmitted successfully as expected. End result were reached Database server (APRS.IS) and were able to use the Web Query Interface check the correction of raw data and Google Map Overlay API successfully.

\section{Conclusions}

We developed the system which be able to track the growth rate of the Forest Industrial where in the rural area that forest locate will not be able to use the internet network because far from Wi-Fi or Internet infrastructure and that why the Packet Radio or APRS is the right choice for this implementation in order to send data over the long distance to the location that have the internet which we use at the Gateway station.

It is very useful for wood industrial and also be able to implement for tracking the Natural resources which can provide security of illegal logging. With this system we are developing where the tree are costly mean to production and plantation planning will be precisely grow with in the correct time frame after the tree batch has been cut down so the new batch will grow back in the correct time in order to maximize production of wooding industrial at the end result of maximum income and profit.

\section{Acknowledgment}

This research of system development was help and supported by FARM-D ASIA Co.Ltd and Industrial Technology assistant program (ITAP) of National Science Technology Development Agency (NSTDA)

\section{References}

(1) Bob Bruninga : "Automatic Packet Reporting System", [online].available : https://www.aprs.org

(2) Addaim, Adnane, Abdelhak Kherras, Abdelhafid Er-Radi El Bachir Zantou, and Ecole Mohammedia d'Ingénieurs : "Design of APRS Network using Low Cost Nanosatellite." In The Seventh IFIP International Conference on Mobile and Wireless Communications Networks (MWCN 2005), Marrakech, Morocco. 2005.
(3) Chaiyasoonthorn S, Hongyim $\mathrm{N}$ and Mitatha, $\mathrm{S}$ : "Building Automatic Packet Report System to report position and radiation data for autonomous robot in the disaster area". In: Control, Automation and Systems (ICCAS), 2015 15th International Conference on. IEEE, 2015. p. 85-88.

(4) Jennifer Jean Jurgens : "The Development of a Carbon-based Resistive Ink Flex Sensor for Use in an Instrumented Glove to Measure Relative Finger Positions\|" Iowa State University, 1995

(5) Ponticelli, Roberto, and P. Gonzalez de Santos : "Full perimeter obstacle contact sensor based on flex sensors." Sensors and Actuators A: Physical147.2 (2008): 441-448.

(6) Shiva Rajagopal and Richard Quan : "Drums Anywhere", [online].available:

https://people.ece.cornell.edu/land/courses/ece4760/Fin alProjects/f2014/svr24_rq32/svr24_rq32/

(7) Borges, L. M., Barroca, N., Velez, F. J., and Lebres, A. $\mathrm{S}$ : "Smart-clothing wireless flex sensor belt network for foetal health monitoring" Pervasive Computing Technologies for Healthcare, 2009. Pervasive Health 2009. 3rd International Conference on. IEEE, 2009.

(8) Chonggang Wang, Tao Jiang and Qian Zhang : "ZigBee ${ }^{\circledR}$ Network Protocols and Applications"

(9) ZigBee specification version 2006 : "ZigBee" document 064112, 2006.

(10)ZigBee Alliance : "ZigBee Specification. Version 1.0" ZigBee Document 053474r06.

(11) Atmel 8-bit Microcontroller with 4/8/16/32 Kbytes Insystem Programmable Flash [online].Available :http://www.atmel.com/devices/atmega328p.aspx

(12) HX1 Radio Matrix VHF Narrow Band FM High Power (300mW)Datasheet,[online]Available: http://www.radiometrix.com/files/additional/hx1.pdf

(13)MicroSat ${ }^{@}$ WX 3 in 1 APRS I-Gate [online]Available http://microsat.com.pl/manual_download.php?file=wx 3in1plus_v141_manual_english.pdf

(14)Dimse, Steve : “APRServe: An internet Backbone for APRS.”[online]Available: https://www.aprs.net/aprserve.doc.html

(15) Gallagher, Tom, and Daniel de Souza. "Five year Growth Measurements for a Eucalyptus Plantation."

(16) APRS.fi APRS Database Query Web-Interface [online].Available: http://aprs.fi/page/about_technical

(17) Weaver and Joshua A. : "An automated system to detect flash floods and alert at-risk communities" Diss. Massachusetts Institute of Technology, 2005. 